\title{
The Young Australian Loneliness Survey: understanding loneliness in adolescence and young adulthood Research summary
}

\section{Are young people lonely?}

Loneliness is a subjective experience of social isolation. It is related to the perceived quality - rather than quantity - of a person's relationships. Loneliness is characterised by feelings such as social disconnection and feeling misunderstood. It is also associated with poorer physical and mental health outcomes and represents an emerging public health problem.

Loneliness can affect anyone, but it may be particularly common among young people. Adolescents and young adults are often well-connected to strong social structures such as families, friends, schools, universities, work environments, social media, sport, and interest groups. However, critical transitions that characterise youth (e.g. leaving school and/or home, beginning higher education or work) can be stressful and compromise these connections and supports, increasing social vulnerability, loneliness, and isolation.

\section{While young people are often well-connected to strong social structures, critical transitions that characterise youth can be stressful and compromise these connections and supports, increasing social vulnerability, loneliness, and isolation}

Little is known about the extent and impacts of loneliness among adolescents and young adults. To address this gap, VicHealth commissioned the Young Australian Loneliness Survey to better understand this important issue in Victorians aged between 12 and 25 years.

\section{Key findings}

- Loneliness is common among adolescents and young adults and is associated with poorer physical and mental health outcomes.

- In the Young Australian Loneliness Survey, a significant proportion of young Victorians reported problematic levels of loneliness. This included one in six adolescents (aged 12-17) and more than one in three young adults (aged 18-25).

- Many were also at risk of social isolation, with young adults again reporting higher levels than adolescents.

- Young women reported higher levels of loneliness, social anxiety and depressive symptoms than young men.

- Lonelier young people are more likely to experience social anxiety and depressive symptoms than those who are less lonely. They are also more likely to report negative affect (negative mood states) and use unhelpful emotional coping strategies.

- While evidence for the effectiveness of interventions that address loneliness is sparse, strategies that focus on promoting good social health may protect young people from loneliness. 


\section{Key findings}

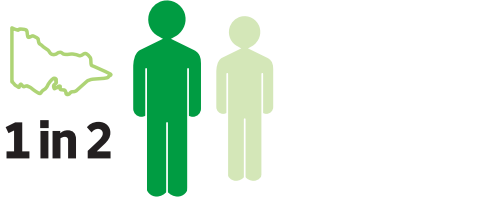

One in two young Victorians reported feeling alone either sometimes or always.

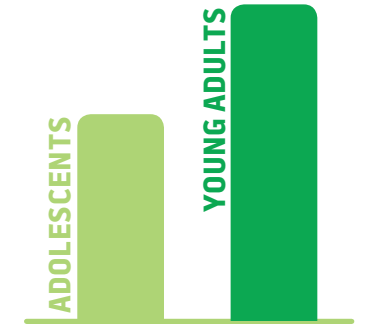

Overall, young adults reported significantly higher rates of loneliness ${ }^{1}$, social isolation ${ }^{2}$, social anxiety ${ }^{3}$, and depressive symptoms ${ }^{4}$ than adolescents.

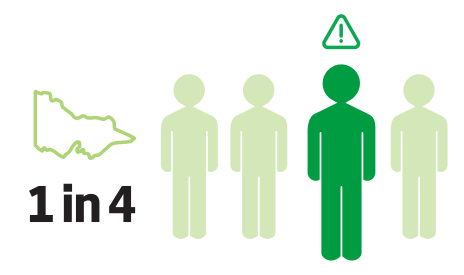

More than one in four young Victorians reported problematic levels of loneliness. ${ }^{1}$

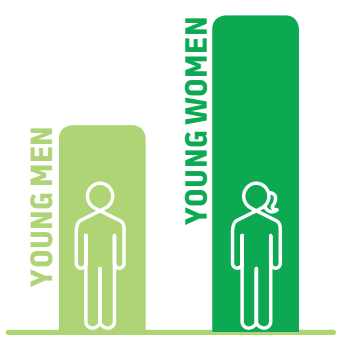

Young women reported higher levels of loneliness, social anxiety and depressive symptoms compared to young men.
YOUNG ADULTS

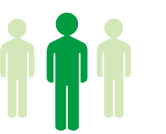

1 in 3
ADOLESCENTS

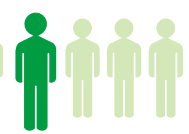

1 in 6

This includes one in six adolescents and more than one in three young adults.

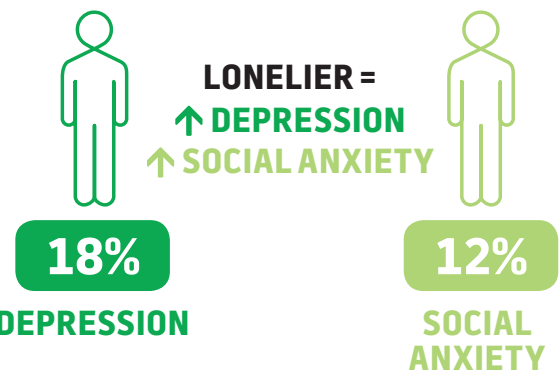

Lonelier young people reporting higher risk for mental ill health (18\% and $12 \%$ for depression and social anxiety respectively).

\section{About this research}

More than 1,500 young Victorians aged $12-25$ completed an online survey between June and July 2019. These included:

- 650 adolescents aged 12-17 years

- 870 young adults aged 18-25 years.

The study aim was to:

- determine how common loneliness and social isolation risk are among 12-25-year-old Victorians

- examine the relationship between loneliness, social isolation risk, and other mental health outcomes.

The survey included measures related to loneliness, risk of social isolation, symptoms of depression and social anxiety, general emotional state (or 'affect'), and how young people tended to respond to negative emotion (i.e. by either reframing their thoughts around the situation or supressing the emotion).

\section{Young adults reported higher levels of loneliness, social isolation, social anxiety and depressive symptoms than adolescents aged 12-17 years}

Greater levels of loneliness in young people were associated with the other factors explored including risk of social isolation, depressive symptoms, and social anxiety. 
Figure 1: Loneliness, social isolation, social anxiety, and depressive symptoms among young Victorians

\begin{tabular}{|c|c|c|c|}
\hline & $\begin{array}{l}\text { Adolescents } \\
\text { (12-17 years) }\end{array}$ & $\begin{array}{l}\text { Young adults } \\
\text { (18-25 years) }\end{array}$ & $\begin{array}{l}\text { Young people } \\
\text { (Overall) }\end{array}$ \\
\hline \multicolumn{4}{|l|}{ Loneliness } \\
\hline Problematic loneliness & $16 \%$ & $37 \%$ & $28 \%$ \\
\hline Feel lonely three or more times a week ${ }^{\mathrm{a}}$ & $13 \%$ & $35 \%$ & $26 \%$ \\
\hline Feel alone sometimes or always & $43 \%$ & $64 \%$ & $55 \%$ \\
\hline Feel they have no one to turn to sometimes or always & $36 \%$ & $55 \%$ & $47 \%$ \\
\hline Lack companionship sometimes or always & $50 \%$ & $62 \%$ & $57 \%$ \\
\hline Feel left out sometimes or always & $48 \%$ & $61 \%$ & $55 \%$ \\
\hline Feel that there are rarely or never people they can talk to & $10 \%$ & $23 \%$ & $17 \%$ \\
\hline \multicolumn{4}{|l|}{ Social isolation } \\
\hline Risk of social isolation & $27 \%$ & $36 \%$ & $32 \%$ \\
\hline See or hear from two or less friends at least once a month & $19 \%$ & $29 \%$ & $25 \%$ \\
\hline Feel close to two or less friends that could be called on for help & $52 \%$ & $55 \%$ & $54 \%$ \\
\hline \multicolumn{4}{|l|}{ Mental health outcomes } \\
\hline Problematic social anxiety symptoms ${ }^{b}$ & $32 \%$ & $58 \%$ & $47 \%$ \\
\hline Problematic depressive symptoms ${ }^{c}$ & $31 \%$ & $64 \%$ & $50 \%$ \\
\hline
\end{tabular}

a This item was taken from the Centre for Epidemiological Studies - Depression (CES-D) scale. The remaining items measuring problematic levels of loneliness are from the revised UCLA loneliness scale (UCLA-LS). Risk of social isolation items are from the Lubben Social Network Scale (LSNS).

b Problematic social anxiety defined as scoring 6 or more on the MINI-Social Phobia Inventory.

c Problematic depressive symptoms defined as score of 20 or more on the CES-D.

\section{What does this mean?}

Loneliness and social isolation - and related mental health outcomes - appear to negatively affect young adults to a greater extent than adolescents. This may suggest that young adults are finding significant life events and transitions (such as leaving school, leaving the family home, and commencing higher education or employment) more problematic than adolescents for whom family, school and other supportive structures remain relatively stable.

Young people around 20 years old have been found to favour the quantity of relationships over the quality of their social interactions which contrasts with their older counterparts at age 30 and $50 .{ }^{5}$ This may explain why they are more vulnerable to feeling lonely, especially if loneliness is a consequence of having fewer meaningful relationships.

Although the relationship between social isolation and loneliness is modest, having fewer contacts with friends and family is associated with loneliness. Other factors associated with loneliness include mental health symptoms such as social anxiety and depression.
At present, there is a lack of evidence for the effectiveness of programs designed to target loneliness in young people specifically. However, strategies that focus on promoting good social health may protect young people from loneliness. Programs that enable young people to better signal an openness to connect with others, enhance their ability to regulate emotions and manage social relationships, and those that can provide safe social opportunities, hold promise. Community-based approaches that increase social connections amongst young people require further investigation.

The findings of this research inform and support VicHealth's Mental Wellbeing Strategy (2019-2023). VicHealth's focus on the mental wellbeing of young people will generate new knowledge about loneliness and empower local governments and community organisations to foster positive social connections among adolescents and young adults.

\section{Strategies that focus on promoting good social health may protect young people from loneliness}




\section{Top Tips for addressing loneliness}

- Act not react. Understand that feeling lonely is normal and it is a signal for you to do something different in your current social relationships.

- Signal. Signal to others your willingness to connect. Simple acts such as smiling and open body language helps others know you are willing to interact.

- Speak. A confidante can help alleviate loneliness, this can relate to a professional person such as a school counsellor or a teacher.

- Focus. Getting more friends may help some but for others it could be easier to focus on improving the quality of a few relationships.

- Find 'your people'. Join a sports team, choir, art class or volunteer - spending time with like-minded people with common interests is a great way to make friends.

- Take the time. Repeated social interactions with others also builds trust.

- Set realistic expectations. Friendships and relationships are dynamic. Your connection with a person wavers over time so don't get down on yourself if you're not seeing certain friends all the time.

- Manage social fears. Your fear of being judged by others may stop you from interacting with people. Seek help to gain more confidence around social interactions to make this easier.

- Manage feeling down. If you think you feel more sad and depressed about your social situation than you should, speak to someone you trust or a health professional.

\footnotetext{
A problematic level of loneliness was defined by a cut off score of 52 or more on the 20-item UCLA Loneliness Scale (Version 3), which includes questions such as 'How often do you feel that there is no one you can turn to?' See also: the Australian Loneliness Report: A survey exploring the loneliness levels of Australians and the impact on their health and wellbeing (2018)

Risk of social isolation was measured by the 6 -item Lubben Social Network Scale, which includes questions such as 'How many friends do you feel close to such that you could call on them for help?'

${ }^{3}$ Problematic social anxiety was defined by a cut off score of 6 or more on the 3-item Mini-Social Phobia Inventory, which prompts responses to statements such as 'I avoid activities in which I am the centre of attention.'

${ }^{4}$ Depressive symptoms were measured by the 20 -item Centre for Epidemiological Studies - Depression measure that assesses feelings of depression in the past 7 days (e.g. how often a person 'felt sad').

${ }^{5}$ Carmichael CL, Reis HT, Duberstein PR (2015) In your 20s it's quantity, in your 30 sit's quality: the prognostic value of social activity across 30 years of adulthood. Psychol Aging 30 (1):95-105. doi:10.1037/pag0000014
}

\section{More information}

- The Young Australian Loneliness Survey (full report)

- VicHealth's Mental Wellbeing Strategy (2019-2023)

\section{Acknowledgements}

This research was undertaken with the Social Health and Wellbeing laboratory at the Iverson Health Innovation Institute, Swinburne University of Technology.

\section{VicHealth}

Victorian Health Promotion Foundation P0 Box 154 Carlton South Victoria 3053 Australia T+61396671333 F+61396671375

vichealth@vichealth.vic.gov.au vichealth.vic.gov.au twitter.com/vichealth facebook.com/vichealth

VicHealth acknowledges the support of the Victorian Government.

(c) VicHealth 2019

October 2019 P-MW-810 